\title{
An inequality related to uncertainty principle in von Neumann algebras
}

\author{
Paolo Gibilisco* and Tommaso Isola ${ }^{\dagger}$
}

November 2, 2018

\begin{abstract}
Recently Kosaki proved in [8] an inequality for matrices that can be seen as a kind of new uncertainty principle. Independently, the same result was proved by Yanagi et al. in [13. The new bound is given in terms of Wigner-Yanase-Dyson informations. Kosaki himself asked if this inequality can be proved in the setting of von Neumann algebras. In this paper we provide a positive answer to that question and moreover we show how the inequality can be generalized to an arbitrary operator monotone function.

2000 Mathematics Subject Classification. Primary 62B10, 94A17; Secondary 46L30, 46L60.

Key words and phrases. Uncertainty principle, Wigner-Yanase-Dyson information, operator monotone functions.
\end{abstract}

\section{Introduction}

If $A, B$ are selfadjoint matrices and $\rho$ is a density matrix, define

$$
\begin{aligned}
\operatorname{Cov}_{\rho}(A, B) & :=\operatorname{Re}\{\operatorname{Tr}(\rho A B)-\operatorname{Tr}(\rho A) \cdot \operatorname{Tr}(\rho B)\} \\
\operatorname{Var}_{\rho}(A) & :=\operatorname{Cov}_{\rho}(A, A) .
\end{aligned}
$$

The uncertainty principle reads as

$$
\operatorname{Var}_{\rho}(A) \operatorname{Var}_{\rho}(B) \geq \frac{1}{4}|\operatorname{Tr}(\rho[A, B])|^{2} .
$$

This inequality can be refined as

$$
\operatorname{Var}_{\rho}(A) \operatorname{Var}_{\rho}(B)-\operatorname{Cov}_{\rho}(A, B)^{2} \geq \frac{1}{4}|\operatorname{Tr}(\rho[A, B])|^{2},
$$

(see [5, 12]). Recently a different uncertainty principle has been found [11, 9, 10, 8, 13]. For $\beta \in(0,1)$ define $\beta$-correlation and $\beta$-information as

$$
\begin{aligned}
\operatorname{Corr}_{\rho, \beta}(A, B) & :=\operatorname{Re}\left\{\operatorname{Tr}(\rho A B)-\operatorname{Tr}\left(\rho^{\beta} A \rho^{1-\beta} B\right)\right\} \\
I_{\rho, \beta}(A) & :=\operatorname{Corr}_{\rho, \beta}(A, A)=\operatorname{Tr}\left(\rho A^{2}\right)-\operatorname{Tr}\left(\rho^{\beta} A \rho^{1-\beta} A\right),
\end{aligned}
$$

where the latter coincides with the Wigner-Yanase-Dyson information. It has been proved that

$$
\operatorname{Var}_{\rho}(A) \operatorname{Var}_{\rho}(B)-\operatorname{Cov}_{\rho}(A, B)^{2} \geq I_{\rho, \beta}(A) I_{\rho, \beta}(B)-\operatorname{Corr}_{\rho, \beta}(A, B)^{2} .
$$

*Dipartimento SEFEMEQ, Facoltà di Economia, Università di Roma "Tor Vergata", Via Columbia 2, 00133 Rome, Italy. Email: gibilisco@volterra.uniroma2.it - URL: http://www.economia.uniroma2.it/sefemeq/professori/gibilisco

†Dipartimento di Matematica, Università di Roma "Tor Vergata", Via della Ricerca Scientifica, 00133 Rome, Italy. Email: isola@mat.uniroma2.it - URL: http://www.mat.uniroma2.it/ isola 
The quantities involved in the previous inequality make a perfect sense in a von Neumann algebra setting (see for example [7]). In ref. [8] Kosaki asked if the inequality (1.1) is true in this more general setting.

In this paper we provide a positive answer to Kosaki question and moreover we show that, once the inequality is formulated in the context of operator monotone functions, the result can be greatly generalized.

\section{Preliminaries}

Denote by $M_{n, s a}$ the space of complex self-adjoint $n \times n$ matrices, and recall that a function $f:(0, \infty) \rightarrow$ $\mathbb{R}$ is said operator monotone if, for any $n \in \mathbb{N}$, any $A, B \in M_{n, s a}$ such that $0 \leq A \leq B$, the inequalities $0 \leq f(A) \leq f(B)$ hold. Then, $f:(0, \infty) \rightarrow \mathbb{R}$ is operator monotone iff for any $A, B \in \mathcal{B}(\mathcal{H})$ such that $0 \leq A \leq B$, it holds $f(A) \leq f(B)$. An operator monotone function is said symmetric if $f(x):=x f\left(x^{-1}\right)$ and normalized if $f(1)=1$. We denote by $\mathfrak{F}$ the class of positive, symmetric, normalized, operator monotone functions.

Examples of operator monotone functions are the so-called Wigner-Yanase-Dyson functions

$$
f_{\beta}(x):=\beta(1-\beta) \frac{(x-1)^{2}}{\left(x^{\beta}-1\right)\left(x^{1-\beta}-1\right)}, \quad \beta \in(0,1) .
$$

Returning to a general $f \in \mathfrak{F}$, we associate to it a function $\tilde{f} \in \mathfrak{F}[2]$ defined by

$$
\tilde{f}(x):=\frac{1}{2}\left((x+1)-(x-1)^{2} \frac{f(0)}{f(x)}\right), x>0 .
$$

For example

$$
\tilde{f}_{\beta}(x)=\frac{1}{2}\left(x^{\beta}+x^{1-\beta}\right) .
$$

Definition 2.1. For $A, B \in M_{n, s a}, f \in \mathfrak{F}$, and $\rho$ a faithful density matrix, define $f$-correlation and $f$-information as

$$
\begin{aligned}
\operatorname{Corr}_{\rho}^{f}(A, B) & :=\operatorname{Re}\left\{\operatorname{Tr}(\rho A B)-\operatorname{Tr}\left(R_{\rho} \tilde{f}\left(L_{\rho} R_{\rho}^{-1}\right)(A) \cdot B\right)\right\}, \\
I_{\rho}^{f}(A) & :=\operatorname{Corr}_{\rho}^{f}(A, A) .
\end{aligned}
$$

Recall that $f$-information is also known as metric adjusted skew information (see 4]). The following generalization of inequality (1.1) is proved in [2].

\section{Theorem 2.2 .}

$$
\operatorname{Var}_{\rho}(A) \operatorname{Var}_{\rho}(B)-\operatorname{Cov}_{\rho}(A, B)^{2} \geq I_{\rho}^{f}(A) I_{\rho}^{f}(B)-\operatorname{Corr}_{\rho}^{f}(A, B)^{2} .
$$

In the next Section we prove that the above inequality holds true in a general von Neumann algebra, thus answering, in particular, the question raised by Kosaki in [8, and recalled above. A different generalization of Theorem 2.2 has been proved in [3].

\section{The main result}

Let $\mathcal{M}$ be a von Neumann algebra, and $\omega$ a normal faithful state on $\mathcal{M}$, and denote by $\mathcal{H}_{\omega}$ and $\xi_{\omega}$ the GNS Hilbert space and vector, and by $S_{\omega}, J_{\omega}$ and $\Delta_{\omega}$ the modular operators associated to $\omega$.

The proof of the main result is divided in a series of Lemmas. In order to deal with unbounded operators, we introduce some sesquilinear forms on $\mathcal{H}_{\omega}$, and take [6] as our standard reference. 
Definition 3.1. Let $f \in \mathfrak{F}$, and define the following sequilinear forms

$$
\begin{aligned}
\mathcal{E}(\xi, \eta) & :=\left\langle\Delta_{\omega}^{1 / 2} \xi, \Delta_{\omega}^{1 / 2} \eta\right\rangle, \\
\mathcal{E}_{1}(\xi, \eta) & :=\mathcal{E}(\xi, \eta)+\langle\xi, \eta\rangle, \\
\mathcal{F}^{f}(\xi, \eta) & :=\left\langle\tilde{f}\left(\Delta_{\omega}\right)^{1 / 2} \xi, \tilde{f}\left(\Delta_{\omega}\right)^{1 / 2} \eta\right\rangle, \\
\mathcal{G}^{f}(\xi, \eta) & :=\frac{1}{2} \mathcal{E}_{1}(\xi, \eta)-\mathcal{F}^{f}(\xi, \eta) .
\end{aligned}
$$

It follows from [6], Example VI.1.13, that $\mathcal{E}, \mathcal{E}_{1}, \mathcal{F}^{f}$ are closed, positive and symmetric sesquilinear forms.

Lemma 3.2. Let $\xi, \eta \in \mathcal{D}\left(\Delta_{\omega}^{1 / 2}\right)$, and $\left\{\xi_{n}\right\},\left\{\eta_{n}\right\} \subset \mathcal{D}\left(\Delta_{\omega}\right)$ be such that $\xi_{n} \rightarrow \xi, \mathcal{E}\left(\xi_{n}-\xi, \xi_{n}-\xi\right) \rightarrow 0$, $n \rightarrow \infty$, and analogously for $\eta_{n}$ and $\eta$. Then

$$
\begin{aligned}
\mathcal{E}(\xi, \eta) & =\lim _{n \rightarrow \infty} \mathcal{E}\left(\xi_{n}, \eta_{n}\right)=\lim _{n \rightarrow \infty}\left\langle\xi_{n}, \Delta_{\omega} \eta_{n}\right\rangle, \\
\mathcal{F}^{f}(\xi, \eta) & =\lim _{n \rightarrow \infty} \mathcal{F}^{f}\left(\xi_{n}, \eta_{n}\right)=\lim _{n \rightarrow \infty}\left\langle\xi_{n}, \tilde{f}\left(\Delta_{\omega}\right) \eta_{n}\right\rangle .
\end{aligned}
$$

Proof. It follows from [6] Theorem VI.2.1 that $\mathcal{D}\left(\Delta_{\omega}\right)$ is a core for $\mathcal{D}(\mathcal{E}) \equiv \mathcal{D}\left(\Delta_{\omega}^{1 / 2}\right)$, so that, from 6 . Theorem VI.1.21, for any $\xi \in \mathcal{D}\left(\Delta_{\omega}^{1 / 2}\right)$ there is $\left\{\xi_{n}\right\} \subset \mathcal{D}\left(\Delta_{\omega}\right)$ such that $\xi_{n} \rightarrow \xi$, and $\mathcal{E}\left(\xi_{n}-\xi, \xi_{n}-\xi\right) \rightarrow 0$, $n \rightarrow \infty$. Then $\mathcal{E}\left(\xi_{n}-\xi_{m}, \xi_{n}-\xi_{m}\right) \rightarrow 0, m, n \rightarrow \infty$. Now observe that $0 \leq \tilde{f}(x) \leq \frac{1}{2}(x+1)$, for $x>0$ [2], so that

$$
\begin{aligned}
\mathcal{F}^{f}\left(\xi_{n}-\xi_{m}, \xi_{n}-\xi_{m}\right) & =\left\langle\tilde{f}\left(\Delta_{\omega}\right)^{1 / 2}\left(\xi_{n}-\xi_{m}\right), \tilde{f}\left(\Delta_{\omega}\right)^{1 / 2}\left(\xi_{n}-\xi_{m}\right)\right\rangle \\
& =\left\langle\xi_{n}-\xi_{m}, \tilde{f}\left(\Delta_{\omega}\right)\left(\xi_{n}-\xi_{m}\right)\right\rangle \\
& \leq \frac{1}{2}\left\langle\xi_{n}-\xi_{m}, \xi_{n}-\xi_{m}\right\rangle+\frac{1}{2}\left\langle\xi_{n}-\xi_{m}, \Delta_{\omega}\left(\xi_{n}-\xi_{m}\right)\right\rangle \\
& =\frac{1}{2}\left\|\xi_{n}-\xi_{m}\right\|+\frac{1}{2} \mathcal{E}\left(\xi_{n}-\xi_{m}, \xi_{n}-\xi_{m}\right) \rightarrow 0, m, n \rightarrow \infty
\end{aligned}
$$

This implies $\xi \in \mathcal{D}\left(\mathcal{F}^{f}\right)$ and $\mathcal{F}^{f}\left(\xi_{n}-\xi, \xi_{n}-\xi\right) \rightarrow 0, n \rightarrow \infty$.

Therefore, if $\xi, \eta \in \mathcal{D}\left(\Delta_{\omega}^{1 / 2}\right)$, and $\left\{\xi_{n}\right\},\left\{\eta_{n}\right\} \subset \mathcal{D}\left(\Delta_{\omega}\right)$ approximate $\xi, \eta$ in the above sense, we obtain, from [6] Theorem VI.1.12, that $\mathcal{F}^{f}(\xi, \eta)=\lim _{n \rightarrow \infty} \mathcal{F}^{f}\left(\xi_{n}, \eta_{n}\right)$, and analogously for $\mathcal{E}$.

\section{Lemma 3.3.}

(i) $\mathcal{D}\left(\mathcal{F}^{f}\right) \supset \mathcal{D}\left(\Delta_{\omega}^{1 / 2}\right)$,

(ii) $\mathcal{G}^{f}$ is a symmetric sesquilinear form on $\mathcal{D}\left(\mathcal{G}^{f}\right) \supset \mathcal{D}\left(\Delta_{\omega}^{1 / 2}\right)$, which is positive on $\mathcal{D}\left(\Delta_{\omega}^{1 / 2}\right)$.

Proof. (i) It follows from the proof of the previous Lemma.

(ii) We only need to prove positivity. To begin with, let $\xi \in \mathcal{D}\left(\Delta_{\omega}\right)$. Then, setting $g(x):=\frac{1}{2}(x+1)-$ $\tilde{f}(x) \geq 0$, for all $x>0$, we have $\mathcal{G}^{f}(\xi, \xi)=\frac{1}{2} \mathcal{E}_{1}(\xi, \xi)-\mathcal{F}^{f}(\xi, \xi)=\frac{1}{2}\langle\xi, \xi\rangle+\frac{1}{2}\left\langle\xi, \Delta_{\omega} \xi\right\rangle-\left\langle\xi, \tilde{f}\left(\Delta_{\omega}\right) \xi\right\rangle=$ $\left\langle\xi, g\left(\Delta_{\omega}\right) \xi\right\rangle \geq 0$.

Moreover, if $\xi \in \mathcal{D}\left(\Delta_{\omega}^{1 / 2}\right)$, and $\xi_{n} \in \mathcal{D}\left(\Delta_{\omega}\right)$ is such that $\xi_{n} \rightarrow \xi$, and $\mathcal{E}\left(\xi_{n}-\xi, \xi_{n}-\xi\right) \rightarrow 0$, then, from Lemma 3.2 it follows $\mathcal{G}^{f}(\xi, \xi)=\lim _{n \rightarrow \infty} \mathcal{G}^{f}\left(\xi_{n}, \xi_{n}\right) \geq 0$.

We can now introduce the main objects of study. In the sequel, we denote by $T \widehat{\in} \mathcal{M}$ the fact that $T$ is a closed, densely defined, linear operator on $\mathcal{H}_{\omega}$, and is affiliated with $\mathcal{M}$.

Definition 3.4. For any $A, B \widehat{\in \mathcal{M}}{ }_{s a}$, such that $\xi_{\omega} \in \mathcal{D}(A) \cap \mathcal{D}(B)$, and any $f \in \mathfrak{F}$, we set $A_{0}:=$ $A-\left\langle\xi_{\omega}, A \xi_{\omega}\right\rangle, B_{0}:=B-\left\langle\xi_{\omega}, B \xi_{\omega}\right\rangle$, and define the bilinear forms

$$
\begin{aligned}
\operatorname{Cov}_{\omega}(A, B) & :=\operatorname{Re}\left\langle A_{0} \xi_{\omega}, B_{0} \xi_{\omega}\right\rangle \\
\operatorname{Var}_{\omega}(A) & :=\operatorname{Cov}_{\omega}(A, A) \\
\operatorname{Corr}_{\omega}^{f}(A, B) & :=\operatorname{Re}\left\langle A_{0} \xi_{\omega}, B_{0} \xi_{\omega}\right\rangle-\operatorname{Re}\left\langle\tilde{f}\left(\Delta_{\omega}\right)^{1 / 2} A_{0} \xi_{\omega}, \tilde{f}\left(\Delta_{\omega}\right)^{1 / 2} B_{0} \xi_{\omega}\right\rangle, \\
I_{\omega}^{f}(A) & :=\operatorname{Corr}_{\omega}^{f}(A, A) .
\end{aligned}
$$


Remark 3.5. Observe that in the matrix case $\omega=\operatorname{Tr}(\rho \cdot)$, for some density matrix $\rho$, and $\Delta_{\omega}=L_{\rho} R_{\rho}^{-1}$, so that the previous Definition is a true generalization of covariance and $f$-correlation in the matrix case.

For the reader's convenience, we prove the following folklore result.

Lemma 3.6. $\mathcal{D}\left(\Delta_{\omega}^{1 / 2}\right)=\left\{T \xi_{\omega}: T \widehat{\in} \mathcal{M}, \xi_{\omega} \in \mathcal{D}(T) \cap \mathcal{D}\left(T^{*}\right)\right\}$.

Proof. (1) Let us first prove that $\mathcal{D}\left(\Delta_{\omega}^{1 / 2}\right) \subset\left\{T \xi_{\omega}: T \widehat{\in} \mathcal{M}, \xi_{\omega} \in \mathcal{D}(T) \cap \mathcal{D}\left(T^{*}\right)\right\}$. Indeed, let $\eta \in \mathcal{D}\left(\Delta_{\omega}^{1 / 2}\right)$, and define the linear operator $T_{0}: x^{\prime} \xi_{\omega} \in \mathcal{N}^{\prime} \xi_{\omega} \mapsto x^{\prime} \eta \in \mathcal{H}_{\omega}$, which is densely defined, and affiliated with $\mathcal{M}$. Let us show that is preclosed: indeed, if $x_{n}^{\prime} \xi_{\omega} \rightarrow 0$, and $x_{n}^{\prime} \eta \rightarrow \zeta$, then, for any $y^{\prime} \in \mathcal{M}^{\prime}$, we get

$$
\begin{aligned}
\left\langle\zeta, y^{\prime} \xi_{\omega}\right\rangle & =\lim _{n \rightarrow \infty}\left\langle x_{n}^{\prime} \eta, y^{\prime} \xi_{\omega}\right\rangle=\lim _{n \rightarrow \infty}\left\langle\eta, x_{n}^{\prime *} y^{\prime} \xi_{\omega}\right\rangle=\lim _{n \rightarrow \infty}\left\langle\eta, S_{\omega}^{*}\left(y^{\prime *} x_{n}^{\prime} \xi_{\omega}\right)\right\rangle \\
& =\lim _{n \rightarrow \infty}\left\langle y^{\prime *} x_{n}^{\prime} \xi_{\omega}, S_{\omega} \eta\right\rangle=\lim _{n \rightarrow \infty}\left\langle x_{n}^{\prime} \xi_{\omega}, y^{\prime} S_{\omega} \eta\right\rangle=0,
\end{aligned}
$$

which shows that $T_{0}$ is preclosed. Let $T_{\eta}:=\overline{T_{0}}$. Then, $T_{\eta} \widehat{\in \mathcal{M}}$, and $T_{\eta} \xi_{\omega}=\eta$. It remains to be proved that $\xi_{\omega} \in \mathcal{D}\left(T_{\eta}^{*}\right)$. Since $S_{\omega} \eta \in \mathcal{D}\left(\Delta_{\omega}^{1 / 2}\right)$, we can also consider $T_{S_{\omega} \eta}$. Let us show that $T_{S_{\omega} \eta} \subset T_{\eta}^{*}$. Indeed, for any $x^{\prime}, y^{\prime} \in \mathcal{M}^{\prime}$, we have

$$
\left\langle T_{S_{\omega} \eta} x^{\prime} \xi_{\omega}, y^{\prime} \xi_{\omega}\right\rangle=\left\langle x^{\prime} S_{\omega} \eta, y^{\prime} \xi_{\omega}\right\rangle=\left\langle S_{\omega} \eta, x^{\prime *} y^{\prime} \xi_{\omega}\right\rangle=\left\langle y^{\prime *} x^{\prime} \xi_{\omega}, \eta\right\rangle=\left\langle x^{\prime} \xi_{\omega}, y^{\prime} \eta\right\rangle=\left\langle x^{\prime} \xi_{\omega}, T_{\eta} y^{\prime} \xi_{\omega}\right\rangle .
$$

Then, $\xi_{\omega} \in \mathcal{D}\left(T_{S_{\omega} \eta}\right) \subset \mathcal{D}\left(T_{\eta}^{*}\right)$, which shows that $\mathcal{D}\left(\Delta_{\omega}^{1 / 2}\right) \subset\left\{T \xi_{\omega}: T \widehat{\in} \mathcal{M}, \xi_{\omega} \in \mathcal{D}(T) \cap \mathcal{D}\left(T^{*}\right)\right\}$.

(2) Let us now prove that $\mathcal{D}\left(\Delta_{\omega}^{1 / 2}\right) \supset\left\{T \xi_{\omega}: T \widehat{\in} \mathcal{M}, \xi_{\omega} \in \mathcal{D}(T) \cap \mathcal{D}\left(T^{*}\right)\right\}$. Indeed, if $T \widehat{\in} \mathcal{M}$ is such that $\xi_{\omega} \in \mathcal{D}(T) \cap \mathcal{D}\left(T^{*}\right)$, we can consider its polar decomposition $T=v|T|$, and let $e_{n}:=\chi_{[0, n]}(|T|)$, $T_{n}:=v|T| e_{n}$, for any $n \in \mathbb{N}$. Since $\xi_{\omega} \in \mathcal{D}(T)$, we have $T_{n} \xi_{\omega}=v e_{n}|T| \xi_{\omega} \rightarrow T \xi_{\omega}$. Moreover, since $\xi_{\omega} \in \mathcal{D}\left(T^{*}\right)$, we have $T_{n}^{*} \xi_{\omega}=|T| e_{n} v^{*} \xi_{\omega}=e_{n} T^{*} \xi_{\omega} \rightarrow T^{*} \xi_{\omega}$. Since $S_{\omega}$ is a closed operator, it follows that $T \xi_{\omega} \in \mathcal{D}\left(S_{\omega}\right)=\mathcal{D}\left(\Delta_{\omega}^{1 / 2}\right)$ [and $S_{\omega} T \xi_{\omega}=T^{*} \xi_{\omega}$ ], which is what we wanted to prove.

Lemma 3.7. For any $A, B \widehat{\in} \mathcal{M}_{s a}$, such that $\xi_{\omega} \in \mathcal{D}(A) \cap \mathcal{D}(B)$, and any $f \in \mathfrak{F}$, we have

(i) $\operatorname{Cov}_{\omega}(A, B)=\frac{1}{2} \operatorname{Re} \varepsilon_{1}\left(A_{0} \xi_{\omega}, B_{0} \xi_{\omega}\right)$ is a positive bilinear form,

(ii) $\operatorname{Corr}_{\omega}^{f}(A, B)=\operatorname{Re}^{f}\left(A_{0} \xi_{\omega}, B_{0} \xi_{\omega}\right)$ is a positive bilinear form.

Proof. (i) Observe that

$$
\begin{aligned}
\left\langle B_{0} \xi_{\omega}, A_{0} \xi_{\omega}\right\rangle & =\left\langle B_{0}^{*} \xi_{\omega}, A_{0}^{*} \xi_{\omega}\right\rangle=\left\langle J_{\omega} \Delta_{\omega}^{1 / 2} B_{0} \xi_{\omega}, J_{\omega} \Delta_{\omega}^{1 / 2} A_{0} \xi_{\omega}\right\rangle \\
& =\left\langle\Delta_{\omega}^{1 / 2} A_{0} \xi_{\omega}, \Delta_{\omega}^{1 / 2} B_{0} \xi_{\omega}\right\rangle=\mathcal{E}\left(A_{0} \xi_{\omega}, B_{0} \xi_{\omega}\right) .
\end{aligned}
$$

The thesis follows from this and the fact that $\mathcal{D}\left(\Delta_{\omega}^{1 / 2}\right)=\left\{T \xi_{\omega}: T \widehat{\in} \mathcal{M}, \xi_{\omega} \in \mathcal{D}(T) \cap \mathcal{D}\left(T^{*}\right)\right\}$.

(ii) It follows from $(i)$ and Lemma 3.3 (ii).

Lemma 3.8. Let $\xi, \eta \in \mathcal{H}_{\omega}, \Delta_{\omega}=\int_{0}^{\infty} t$ de(t), and define, for $\Omega$ a Borel subset of $[0, \infty), \mu_{\xi \eta}(\Omega):=$ $\operatorname{Re}\langle\xi, e(\Omega) \eta\rangle$, and

$$
\mu:=\mu_{\xi \xi} \otimes \mu_{\eta \eta}+\mu_{\eta \eta} \otimes \mu_{\xi \xi}-2 \mu_{\xi \eta} \otimes \mu_{\xi \eta} .
$$

Then, $\mu$ is a bounded positive Borel measure on $[0, \infty)^{2}$.

Proof. Let $\Omega_{1}, \Omega_{2}$ be Borel subsets of $[0, \infty)$, and set $e_{j}:=e\left(\Omega_{j}\right), j=1,2$. Observe that $\mid \operatorname{Re}\left\langle\xi, e_{1} \eta\right\rangle$. $\operatorname{Re}\left\langle\xi, e_{2} \eta\right\rangle \mid \leq\left\|e_{1} \xi\right\| \cdot\left\|e_{1} \eta\right\| \cdot\left\|e_{2} \xi\right\| \cdot\left\|e_{2} \eta\right\|$, so that

$$
\mu\left(\Omega_{1} \times \Omega_{2}\right) \geq\left\|e_{1} \xi\right\|^{2} \cdot\left\|e_{2} \eta\right\|^{2}+\left\|e_{2} \xi\right\|^{2} \cdot\left\|e_{1} \eta\right\|^{2}-2\left\|e_{1} \xi\right\| \cdot\left\|e_{1} \eta\right\| \cdot\left\|e_{2} \xi\right\| \cdot\left\|e_{2} \eta\right\| \geq 0 .
$$

The thesis follows by standard measure theoretic arguments.

Theorem 3.9. For any $A, B \widehat{\in} \mathcal{M}_{s a}$, such that $\xi_{\omega} \in \mathcal{D}(A) \cap \mathcal{D}(B)$, and any $f \in \mathfrak{F}$, we have

$$
\operatorname{Var}_{\omega}(A) \operatorname{Var}_{\omega}(B)-\operatorname{Cov}_{\omega}(A, B)^{2} \geq I_{\omega}^{f}(A) I_{\omega}^{f}(B)-\operatorname{Corr}_{\omega}^{f}(A, B)^{2} .
$$


Proof. Set

$$
\begin{aligned}
G(A, B):= & \operatorname{Var}_{\omega}(A) \operatorname{Var}_{\omega}(B)-\operatorname{Cov}_{\omega}(A, B)^{2}-I_{\omega}^{f}(A) I_{\omega}^{f}(B)+\operatorname{Corr}_{\omega}^{f}(A, B)^{2} \\
\stackrel{(a)}{=} & \frac{1}{2} \varepsilon_{1}\left(A_{0} \xi_{\omega}, A_{0} \xi_{\omega}\right) \cdot \frac{1}{2} \varepsilon_{1}\left(B_{0} \xi_{\omega}, B_{0} \xi_{\omega}\right)-\left(\frac{1}{2} \operatorname{Re} \varepsilon_{1}\left(A_{0} \xi_{\omega}, B_{0} \xi_{\omega}\right)\right)^{2} \\
& -\left(\frac{1}{2} \varepsilon_{1}\left(A_{0} \xi_{\omega}, A_{0} \xi_{\omega}\right)-\mathcal{F}^{f}\left(A_{0} \xi_{\omega}, A_{0} \xi_{\omega}\right)\right)\left(\frac{1}{2} \varepsilon_{1}\left(B_{0} \xi_{\omega}, B_{0} \xi_{\omega}\right)-\mathcal{F}^{f}\left(B_{0} \xi_{\omega}, B_{0} \xi_{\omega}\right)\right) \\
& +\left(\frac{1}{2} \operatorname{Re} \mathcal{E}_{1}\left(A_{0} \xi_{\omega}, B_{0} \xi_{\omega}\right)-\operatorname{Re} \mathcal{F}^{f}\left(A_{0} \xi_{\omega}, B_{0} \xi_{\omega}\right)\right)^{2} \\
= & \frac{1}{2} \varepsilon_{1}\left(A_{0} \xi_{\omega}, A_{0} \xi_{\omega}\right) \cdot \mathcal{F}^{f}\left(B_{0} \xi_{\omega}, B_{0} \xi_{\omega}\right)+\frac{1}{2} \mathcal{F}^{f}\left(A_{0} \xi_{\omega}, A_{0} \xi_{\omega}\right) \cdot \mathcal{E}_{1}\left(B_{0} \xi_{\omega}, B_{0} \xi_{\omega}\right) \\
& -\mathcal{F}^{f}\left(A_{0} \xi_{\omega}, A_{0} \xi_{\omega}\right) \cdot \mathcal{F}^{f}\left(B_{0} \xi_{\omega}, B_{0} \xi_{\omega}\right)-\operatorname{Re} \mathcal{E}_{1}\left(A_{0} \xi_{\omega}, B_{0} \xi_{\omega}\right) \cdot \operatorname{Re} \mathcal{F}^{f}\left(A_{0} \xi_{\omega}, B_{0} \xi_{\omega}\right) \\
& +\left(\operatorname{Re} \mathcal{F}^{f}\left(A_{0} \xi_{\omega}, B_{0} \xi_{\omega}\right)\right)^{2},
\end{aligned}
$$

where in (a) we have used Lemma 3.7 Let us now introduce the function, for $\xi, \eta \in \mathcal{D}\left(\Delta_{\omega}^{1 / 2}\right)$,

$H(\xi, \eta):=\frac{1}{2} \varepsilon_{1}(\xi, \xi) \cdot \mathcal{F}^{f}(\eta, \eta)+\frac{1}{2} \mathcal{F}^{f}(\xi, \xi) \cdot \mathcal{E}_{1}(\eta, \eta)-\mathcal{F}^{f}(\xi, \xi) \cdot \mathcal{F}^{f}(\eta, \eta)-\operatorname{Re} \varepsilon_{1}(\xi, \eta) \cdot \operatorname{Re} \mathcal{F}^{f}(\xi, \eta)+\left(\operatorname{Re} \mathcal{F}^{f}(\xi, \eta)\right)^{2}$,

and recall that $\mathcal{D}\left(\Delta_{\omega}^{1 / 2}\right)=\left\{T \xi_{\omega}: T \widehat{\in} \mathcal{M}, \xi_{\omega} \in \mathcal{D}(T) \cap \mathcal{D}\left(T^{*}\right)\right\}$, so that, if $A, B$ are as in the statement of the Theorem, we obtain $G(A, B)=H\left(A_{0} \xi_{\omega}, B_{0} \xi_{\omega}\right)$, and to prove the theorem it suffices to show that $H(\xi, \eta) \geq 0$, for all $\xi, \eta \in \mathcal{D}\left(\Delta_{\omega}^{1 / 2}\right)$. Observe that, for $\xi, \eta \in \mathcal{D}\left(\Delta_{\omega}\right)$, we get

$$
\begin{aligned}
& H(\xi, \eta)=\frac{1}{2}\left\langle\xi,\left(1+\Delta_{\omega}\right) \xi\right\rangle \cdot\left\langle\eta, \tilde{f}\left(\Delta_{\omega}\right) \eta\right\rangle+\frac{1}{2}\left\langle\eta,\left(1+\Delta_{\omega}\right) \eta\right\rangle \cdot\left\langle\xi, \tilde{f}\left(\Delta_{\omega}\right) \xi\right\rangle \\
& -\left\langle\xi, \tilde{f}\left(\Delta_{\omega}\right) \xi\right\rangle \cdot\left\langle\eta, \tilde{f}\left(\Delta_{\omega}\right) \eta\right\rangle-\operatorname{Re}\left\langle\xi,\left(1+\Delta_{\omega}\right) \eta\right\rangle \cdot \operatorname{Re}\left\langle\xi, \tilde{f}\left(\Delta_{\omega}\right) \eta\right\rangle+\left(\operatorname{Re}\left\langle\xi, \tilde{f}\left(\Delta_{\omega}\right) \eta\right\rangle\right)^{2} \\
& \stackrel{(b)}{=} \frac{1}{2} \int_{0}^{\infty}(s+1) d \mu_{\xi \xi}(s) \int_{0}^{\infty} \tilde{f}(t) d \mu_{\eta \eta}(t)+\frac{1}{2} \int_{0}^{\infty} \tilde{f}(s) d \mu_{\xi \xi}(s) \int_{0}^{\infty}(t+1) d \mu_{\eta \eta}(t) \\
& -\int_{0}^{\infty} \tilde{f}(s) d \mu_{\xi \xi}(s) \int_{0}^{\infty} \tilde{f}(t) d \mu_{\eta \eta}(t)-\frac{1}{2} \int_{0}^{\infty}(s+1) d \mu_{\xi \eta}(s) \int_{0}^{\infty} \tilde{f}(t) d \mu_{\xi \eta}(t) \\
& -\frac{1}{2} \int_{0}^{\infty} \tilde{f}(s) d \mu_{\xi \eta}(s) \int_{0}^{\infty}(t+1) d \mu_{\xi \eta}(t)-\int_{0}^{\infty} \tilde{f}(s) d \mu_{\xi \eta}(s) \int_{0}^{\infty} \tilde{f}(t) d \mu_{\xi \eta}(t) \\
& \stackrel{(c)}{=} \frac{1}{2} \int_{[0, \infty)^{2}}((s+1) \tilde{f}(t)+(t+1) \tilde{f}(s)-2 \tilde{f}(s) \tilde{f}(t)) d \mu_{\xi \xi} \otimes \mu_{\eta \eta}(s, t) \\
& -\frac{1}{2} \int_{[0, \infty)^{2}}((s+1) \tilde{f}(t)+(t+1) \tilde{f}(s)-2 \tilde{f}(s) \tilde{f}(t)) d \mu_{\xi \eta} \otimes \mu_{\xi \eta}(s, t) \\
& \stackrel{(d)}{=} \frac{1}{4} \iint_{[0, \infty)^{2}}((s+1) \tilde{f}(t)+(t+1) \tilde{f}(s)-2 \tilde{f}(s) \tilde{f}(t)) d \mu(s, t),
\end{aligned}
$$

where we used in $(b)$ notation as in Lemma 3.8 in $(c)$ Fubini-Tonelli Theorem, and in $(d)$ the symmetries of the first integrand and notation as in Lemma 3.8.

Since $\mu$ is a positive measure, and

$$
(s+1) \tilde{f}(t)+(t+1) \tilde{f}(s)-2 \tilde{f}(s) \tilde{f}(t)=(s+1-\tilde{f}(s)) \tilde{f}(t)+(t+1-\tilde{f}(t)) \tilde{f}(s) \geq 0,
$$

we obtain $H(\xi, \eta) \geq 0$, for any $\xi, \eta \in \mathcal{D}\left(\Delta_{\omega}\right)$.

It follows from Lemma 3.2 that, for any $\xi, \eta \in \mathcal{D}\left(\Delta_{\omega}^{1 / 2}\right)$, we have $H(\xi, \eta)=\lim _{n \rightarrow \infty} H\left(\xi_{n}, \eta_{n}\right) \geq 0$, which ends the proof.

\section{References}

[1] P. Gibilisco, T. Isola. Uncertainty principle and quantum Fisher information, Ann. Inst. Stat. Math, 59 (2007), 147-159. 
[2] P. Gibilisco, D. Imparato, T. Isola. Uncertainty principle and quantum Fisher information, II, J. Math. Phys., 48 (2007), 072109.

[3] P. Gibilisco, D. Imparato, T. Isola. A Robertson-type uncertainty principle and quantum Fisher information, Lin. Alg. Appl., to appear, arXiv:0707.1231.

[4] F. Hansen. Metric adjusted skew information, preprint 2006, arXiv:math-ph/0607049v3.

[5] W. Heisenberg. Über den anschaulichen inhalt der quantentheoretischen kinematik und mechanik, Zeitschrift für Physik, 43 (1927), 172-198.

[6] T. Kato. Perturbation theory for linear operators, Springer, New York, 1966.

[7] H. Kosaki. Interpolation theory and the Wigner-Yanase-Dyson-Lieb concavity, Comm. Math. Phys., 87 (1982/83), 315-329.

[8] H. Kosaki. Matrix trace inequality related to uncertainty principle, Internat. J. Math., 16 (2005), 629-645.

[9] S. Luo, Q. Zhang. On skew information, IEEE Trans. Inform. Theory, 50 (2004), 1778-1782.

[10] S. Luo, Q. Zhang. Correction to "On skew information", IEEE Trans. Inform. Theory, 51 (2005), 4432.

[11] S. Luo, Z. Zhang. An informational characterization of Schrödinger's uncertainty relations, J. Statist. Phys., 114 (2004), 1557-1576.

[12] E. Schrödinger. About Heisenberg uncertainty relation (original annotation by Angelow A. and Batoni M. C.), Bulgar. J. Phys., 26 (2000), 193-203. Translation of Proc. Prussian Acad. Sci. Phys. Math. Sect., 19 (1930), 296-303.

[13] K. Yanagi, S. Furuichi, K. Kuriyama. A generalized skew information and uncertainty relation, IEEE Trans. Inform. Theory, 51 (2005), 4401-4404. 